\title{
Prediction of key genes in ovarian cancer treated with decitabine based on network strategy
}

\author{
YU-ZHEN WANG ${ }^{1}$ and SHENG-CHUN QIU ${ }^{2}$ \\ ${ }^{1}$ Department of Pharmacy, Sir Run Run Shaw Hospital, School of Medicine, Zhejiang University, Hangzhou, Zhejiang 310016; \\ ${ }^{2}$ Department of Nursing, Zhejiang Provincial People's Hospital, Xiacheng, Hangzhou, Zhejiang 310014, P.R. China
}

Received December 17, 2015; Accepted January 26, 2016

DOI: 10.3892/or.2016.4697

\begin{abstract}
The objective of the present study was to predict key genes in ovarian cancer before and after treatment with decitabine utilizing a network approach and to reveal the molecular mechanism. Pathogenic networks of ovarian cancer before and after treatment were identified based on known pathogenic genes (seed genes) and differentially expressed genes (DEGs) detected by Significance Analysis of Microarrays (SAM) method. A weight was assigned to each gene in the pathogenic network and then candidate genes were evaluated. Topological properties (degree, betweenness, closeness and stress) of candidate genes were analyzed to investigate more confident pathogenic genes. Pathway enrichment analysis for candidate and seed genes were conducted. Validation of candidate gene expression in ovarian cancer was performed by reverse transcriptase-polymerase chain reaction (RT-PCR) assays. There were 73 nodes and 147 interactions in the pathogenic network before treatment, while 47 nodes and 66 interactions after treatment. A total of 32 candidate genes were identified in the before treatment group of ovarian cancer, of which 16 were rightly candidate genes after treatment and the others were silenced. We obtained 5 key genes $(P I K 3 R 2$, $C C N B 1, I L 2, I L 1 B$ and $C D C 6)$ for decitabine treatment that were validated by RT-PCR. In conclusion, we successfully identified 5 key genes (PIK3R2, CCNB1,IL2,IL1B and CDC6) and validated them, which provides insight into the molecular mechanisms of decitabine treatment and may be potential pathogenic biomarkers for the therapy of ovarian cancer.
\end{abstract}

\section{Introduction}

Ovarian cancer is the ninth most common cancer among women and the fifth leading cause of cancer-related death among women with recent statistics suggesting that 1 in

Correspondence to: Dr Sheng-Chun Qiu, Department of Nursing, Zhejiang Provincial People's Hospital, 158 Shangtang Road, Xiacheng, Hangzhou, Zhejiang 310014, P.R. China

E-mail: shengchunqiu2015@yeah.net

Key words: ovarian cancer, network, gene, pathogenic, candidate, seed, reverse transcriptase-polymerase chain reaction
71 women will develop ovarian cancer $(1,2)$. Approximately $70 \%$ of ovarian cancer cases are diagnosed at a late stage and therefore are poorly treatable (3). Although the current standard treatment for ovarian cancer involving the use of paclitaxel and carboplatin after aggressive surgical cytoreduction usually results in multiyear survival, prolonged use of platinum-based chemotherapy often induces drug resistance, which causes ovarian cancer relapse and eventually the death of patients (4). Such knowledge may translate into the development of new targeted strategies. In addition, since ovarian cancer is considered to be a heterogeneous group of diseases with distinct gene expression profiles, it is likely that the focus should be towards the development of new targeted therapies capable of exploiting the molecular and genetic characteristics of ovarian cancer (5). Therefore, it is necessary to understand the pathogenesis of ovarian cancer by dissecting the components involved in the pathogenic procedure, i.e. pathogenic genes.

The pathogenic genes can be identified in the laboratory by techniques, such as gene knockout or silencing, however, the pathogenic gene list is far from complete and it is a painful process to identify pathogenic genes in the laboratory considering the genome size and time-consuming experiments (6). In contrast, computational methods can provide alternative strategies for this issue, for instance, high throughput techniques. Traditionally, studies tend to regard differentially expressed genes (DEGs) between normal and disease samples as biomarkers and pathogenic genes, but, DEGs alone may lead to false positives while identifying key genes involved in disease procedure since some genes are not involved in the pathway of pathogenic genes even though they show significant expression change (7). In the meantime, studies have shown that the most significant genes obtained from different studies for a particular cancer are typically inconsistent (8). To overcome this issue, one could evaluate pathogenic genes for disease-association using a network strategy (9).

5-Aza-2'-deoxycytidine (decitabine) is a prodrug that requires metabolic activation by deoxycytidine kinase, an active inhibitor in the triphosphate form (10). DNA polymerase catalyzes the insertion of the phosphorylated form of decitabine into DNA, and the presence of decitabine in place of the 5-methylcytosine in DNA leads to the inactivation of DNA methyltransferase inducing a re-expression of the silenced genes (11). It has been demonstrated that decitabine produces 
variable antitumor response rates in patients with solid tumors that may be leveraged clinically with identification of a predictive biomarker (12). For instance, decitabine is an effective therapy for myelodysplastic syndromes (MDS) and for acute myeloid leukemia (AML) (13). Moreover, its role in the treatment of ovarian cancer has been defined in regards to the fact that epigenetic therapy upregulates the expression of imprinted tumor suppressors (14). Hence, more and more research has focused on ovarian cancer treatment with decitabine, while the molecular mechanisms of this drug remain unclear.

Therefore, in the present study, we employed a network approach to predict key genes which are potentially silenced genes for ovarian cancer before and after treatment with decitabine. The network approach was based on a pathogenic network that derived from a protein-protein interaction (PPI) network, DEGs and known pathogenic genes (seed genes), to identify candidate genes and silenced genes. Subsequently, topological properties and pathway enrichment analysis were performed for candidate genes. By combining weight values and topological properties of candidate genes and silenced genes before and after treatment with decitabine, we obtained key genes and validated key genes by reverse transcriptasepolymerase chain reaction (RT-PCR) assays.

\section{Materials and methods}

Gene expression data. In the present study, the microarray gene expression profile of ovarian cancer with accession no. E-GEOD-25429 (15) was downloaded from ArrayExpress database. E-GEOD-25429 was comprised of 91 samples (4 normal controls, 43 ovarian cancer samples and 41 ovarian cancer samples treated with decitabine), and deposited on two platforms, A-AFFY-44-Affymetrix GeneChip Human Genome U133 Plus 2.0 [HG-U133_Plus_2] and A-AFFY-113-Affymetrix GeneChip HT Human Genome U133A [HT_HG-U133A]. When mapping the probes to genes according to the platforms, a total of 20,107 and 12,494 genes were obtained, respectively. To avoid batch effects from the different platforms, we took the intersections of two platforms as the gene expression profile which consisted of 12,493 genes for further analysis.

Detection of DEGs. To determine expression changes between normal controls and ovarian cancer before and after treatment with decitabine while accounting for the enormous number of genes, Significance Analysis of Microarrays (SAM) (16), which assigns a score to each gene on the basis of the change in gene expression relative to the standard deviation of repeated measurements, was utilized in the present study. We divided the samples into two conditions, condition 1 (normal controls vs. ovarian cancer before treatment) and condition 2 (normal controls vs. ovarian cancer after treatment with decitabine). By conducting a set of gene-specific $t$-tests among two conditions, genes with statistically significant changes in expression were identified based on SAM. Taking condition 1 as an example, the relative difference $d(i)$ in gene expression was defined as following:

$$
d(i)=\frac{\overline{X_{M}}(i)-\overline{X_{N}}(i)}{s(i)+s_{o}}
$$

\section{Where $\overline{X_{M}}(i)$ and $\overline{X_{N}}(i)$}

are defined as the average levels of expression for gene $i$ in normal and ovarian cancer, respectively. $s(i)$ is the standard deviation of repeated expression measurements. The value for $s_{0}$ was chosen to minimize the coefficient of variation.

To identify significant differentially expressed genes further, genes were ranked in descending order of $d(i)$ values, so that $d(1)$ was the largest relative difference, $d(2)$ was the second largest relative difference, and $d(i)$ was the $i$ th largest relative difference. Meanwhile $d t(i)$ was the $i$ th largest relative difference for permutation $t$. The expected relative difference, $d_{E}(i)$, was defined as the average over all permutations, $d_{E}(i)=\left(\sum t d t(i)\right) / \mathrm{n}$. For the vast majority of genes, $d(i) \cong d_{E}(i)$, but some genes were represented by points displaced from the $d(i)=d_{E}(i)$ line by a distance greater than a threshold $\Delta$. As $\Delta$ decreased, the number of genes called significant by SAM increased, the $\Delta$ value for condition 1 and condition 2 was 3.600 and 3.436 , separately.

Identification of pathogenic network. There are some genes that have been identified as pathogenic genes of ovarian cancer in Online Mendelian Inheritance in Man (OMIM) database, an online catalog of human genes and genetic disorders (17). In the present study, a total of 87 genes were found, which were also called as known pathogenic genes. Taking the intersection with the gene expression profile, we obtained 82 intersected genes and defined them as seed genes (Table I).

Meanwhile, we recruited human PPI from the Search Tool for the Retrieval of Interacting Genes/Proteins (STRING) (18), and interactions with score $>0.5$ were kept as the background PPI network. Subsequently, a network was extracted from the background PPI network that included genes that interacted with seed genes, where the genes were further required to be DEGs of ovarian cancer before (condition 1) and after (condition 2) treatment with decitabine. Therefore, genes in the sub-network were more possibly pathogenic genes. Furthermore, a smaller sub-network that consisted of genes interacting with at least two seed genes was extracted from the previous network and were regarded as the pathogenic network, where the genes in the pathogenic network were believed to be correlated to pathogenesis.

Ranking of the pathogenic genes. To facilitate the biologists to select more confident pathogenic genes from our predictions, each gene was assigned a weight value based on the interactions and co-expression with seed genes, where a gene was more confident to be a pathogenic gene if it interacted and was co-expressed with more seed genes (6). The co-expression was evaluated by Pearson correlation coefficients (PCC) (19) between our predicted pathogenic and seed genes. The weight for gene $x, W(x)$, was calculated as following:

$$
W(x)=\sum_{y \in S} P C C(x, y) \times I(x, y)
$$

where $S$ is the set of seed genes, $\operatorname{PCC}(x, y)$ is the correlation coefficient between gene $x$ and gene $y$, and $I(x, y)$ is an indication function, where $I(x, y)=1$ if protein $x$ interacted with protein $y$ and $I(x, y)=0$ otherwise. The weight of each predicted pathogenic gene could illustrate the correlation 
Table I. Seed genes of ovarian cancer.

\begin{tabular}{|c|c|c|c|c|c|c|c|}
\hline ID & Gene & ID & Gene & ID & Gene & ID & Gene \\
\hline 1 & $M U C 1$ & 22 & TNFRSF $1 B$ & 43 & SERBPI & 64 & CLIC4 \\
\hline 2 & $T P M 3$ & 23 & RASAL2 & 44 & VCAM1 & 65 & RNASEL \\
\hline 3 & UCHL5 & 24 & PEA15 & 45 & $G A D D 45 A$ & 66 & EPHA2 \\
\hline 4 & MDM4 & 25 & CHI3LI & 46 & $C D 34$ & 67 & MASP2 \\
\hline 5 & $T P 73$ & 26 & SELENBP 1 & 47 & NTRK1 & 68 & $H S D 3 B 2$ \\
\hline 6 & SHCl & 27 & $R W D D 3$ & 48 & $C R P$ & 69 & $H S D 3 B 1$ \\
\hline 7 & MTHFR & 28 & RUNX3 & 49 & $W N T 2 B$ & 70 & $P A R P 1$ \\
\hline 8 & $P B X 1$ & 29 & $N A S P$ & 50 & $\mathrm{KCNHI}$ & 71 & $A S P M$ \\
\hline 9 & EXO1 & 30 & $R A D 54 L$ & 51 & EFNAI & 72 & $J U N$ \\
\hline 10 & AKT3 & 31 & $I K B K E$ & 52 & RORI & 73 & $S L C 2 A 1$ \\
\hline 11 & $F G R$ & 32 & BCL10 & 53 & $F C N 3$ & 74 & $R A B 25$ \\
\hline 12 & VTCN1 & 33 & $D P Y D$ & 54 & FASLG & 75 & CHD5 \\
\hline 13 & DESI2 & 34 & PTGS2 & 55 & HDAC1 & 76 & NES \\
\hline 14 & COLIIA1 & 35 & PTAFR & 56 & ILIO & 77 & $S F N$ \\
\hline 15 & MTOR & 36 & $C D 247$ & 57 & LPAR3 & 78 & TACSTD2 \\
\hline 16 & KIF14 & 37 & $N G F$ & 58 & LIN28A & 79 & S100A6 \\
\hline 17 & THEMIS2 & 38 & $P R D X 1$ & 59 & S100A4 & 80 & PRDX6 \\
\hline 18 & GSTM1 & 39 & $D V L 1$ & 60 & $Y B X 1$ & 81 & LAMTOR5 \\
\hline 19 & $E 2 F 2$ & 40 & MCL1 & 61 & KISSI & 82 & MLLT11 \\
\hline 20 & $A D S S$ & 41 & $F 3$ & 62 & DIRAS3 & & \\
\hline 21 & KCNK2 & 42 & EPHXI & 63 & $T G F B 2$ & & \\
\hline
\end{tabular}

between this gene and the seed genes. The higher the weight of one gene, the more possible the gene was involved in the pathogenic procedure. In addition, we defined the potential pathogenic genes not seed genes as candidate genes of ovarian cancer.

Properties of the pathogenic network. For the purpose of investigating the possible roles of candidate genes, topological properties of nodes in the pathogenic network were explored, including degree, betweenness, closeness and stress. For an undirected network $G=(V, E)$, where $V$ is the set of vertices representing nodes in the network, and $E$ is the set of edges representing the relationships between the actors. A path from node $s$ to $t$ was defined as a sequence of edges and the length of a path was the sum of the weights of edges. We used $d(s, t)$ to denote the distance between $s$ and $t$ (the minimum length of any path connecting $s$ and $t$ in $G$ ). Let us denote the total number of shortest paths between vertices $s$ and $t$ by $\sigma_{s t}$, and the number passing through node $v$ by $\sigma_{s t}(v)$.

Degree. Degree is a simple local measure, based on the notion of neighborhood. It quantifies the local topology of each gene by summing up the number of its adjacent genes (20). The degree $D(v)$ of a node $v$ was defined as:

$$
D(v)=\sum_{j} a_{v j}
$$

Betweenness centrality. Betweenness centrality, $C_{B}(v)$, is a shortest paths enumeration-based metric in graphs for determining how the neighbors of a node are interconnected, and is considered as the ratio of the node in the shortest path between two other nodes $(21)$, in consequence $C_{B}(v) \in[0,1]$. It was calculated as follows:

$$
C_{B}(v)=\sum_{s \neq v \neq t \in N} \frac{\sigma_{s t}(v)}{\sigma_{s t}}
$$

Closeness centrality. Closeness centrality, $C_{c}(v)$, is a measure of the average length of the shortest paths to access all other proteins in the network (22). It was defined as the reciprocal of the average shortest path length:

$$
C_{C}(v)=\frac{1}{\sum_{t \in N} d(s, t)}
$$

Stress. This index computes the number of nodes in the shortest path between two other nodes (23). If a node was stressed, it would be traversed by a high number of shortest paths. The stress, $C_{s}(v)$ was defined as:

$$
C_{S}(v)=\sum_{s \neq v \in N} \sum_{t \neq v \in N} \sigma_{s t}(v)
$$

Pathway enrichment analysis of candidate genes. Kyoto Encyclopedia of Genes and Genomes (KEGG) pathway enrichment analysis for candidate and seed genes were performed based on the Database for Annotation, Visualization and Integrated Discovery (DAVID) (24). In addition, pathways which met the criterion $\mathrm{P}<0.01$ were selected according to Expression Analysis Systematic Explorer (EASE) test implemented in DAVID (25). The calculating formula of EASE is shown as follows:

$$
\mathrm{P}=\frac{\left(\begin{array}{c}
a+b \\
a
\end{array}\right)\left(\begin{array}{c}
c+d \\
c
\end{array}\right)}{\left(\begin{array}{c}
n \\
a+c
\end{array}\right)}
$$


Table II. Primer sequences for the five genes validated by RT-PCR.

Primers $\left(5^{\prime}-3^{\prime}\right)$

\begin{tabular}{lllc}
\cline { 2 - 3 } Gene & \multicolumn{1}{c}{ Forward } & \multicolumn{1}{c}{ Reverse } & Length (bp) \\
\hline PIK3R2 & ATGGCACCTTCCTAGTCCGAGA & CTCTGAGAAGCCATAGTGCCCA & 127 \\
$C C N B 1$ & GACCTGTGTCAGGCTTTCTCTG & GGTATTTTGGTCTGACTGCTTGC & 120 \\
$I L 2$ & AGAACTCAAACCTCTGGAGGAAG & GCTGTCTCATCAGCATATTCACAC & 152 \\
$I L 1 B$ & TCAGCATTAACATGCGTGCTTTCC & CTTTATATCCTATGAATGAGCCATCTG & 104 \\
$C D C 6$ & CAGTAGACACAAAACAGGCTCAG & TGTCGGATCTCCCTCACCAATG & 123 \\
$\beta$-actin & CTCCATCCTGGCCTCGCTGT & GCTGTCACCTTCACCGTTCC & 268 \\
\hline
\end{tabular}

Of which $a=a^{\prime}-1, a^{\prime}$ is the gene number of one gene set in the gene lists; $a^{\prime}+b$ is the number of genes in the gene list including at least one gene set; $a^{\prime}+c$ is the gene number of one gene list in the background genes; $n=a^{\prime}+b+c+d$ is the number of background genes in EASE.

Validation of candidate genes by RT-PCR. RT-PCR assays were carried out to validate key genes. Total RNA was prepared from ovarian cancer cell line A2780 before and after treatment of decitabine, and 10 ovarian cancer patient tissues using TRIzol reagent (Invitrogen, Carlsbad, CA, USA). In the present study, ovarian cancer cell line A2780 was kindly provided by the Cancer Center, Qilu Hospital of Shandong University (Jinan, China). Cells were cultivated in Dulbecco's modified Eagle's medium (DMEM)/F-12 containing 10\% fetal bovine serum (FBS) (Gibco Life Technologies, Carlsbad, CA, USA), and antibiotics (100 U/ml penicillin $\mathrm{G}, 100 \mu \mathrm{g} / \mathrm{ml}$ streptomycin) and $250 \mathrm{ng} / \mathrm{ml}$ fungizone (Roth, Karlsruhe, Germany) at $37^{\circ} \mathrm{C}$ in a humidified incubator with $5 \% \mathrm{CO}_{2}$ atmosphere (Shanghai Sumsung Experimental Instrument Co., Ltd., Shanghai, China). When the cultures reached confluency ( 6 days), the cells were treated with $0.05 \%$ trypsin $/ 1 \mathrm{mM}$ EDTA for $5 \mathrm{~min}$ at $37^{\circ} \mathrm{C}$. Subsequently, the cell suspension was diluted with DMEM/F-12 supplemented with $10 \%$ FBS to a concentration of $2 \times 10^{5}$ cells $/ \mathrm{ml}$, and plated in 12-well culture plates ( $1 \mathrm{ml} /$ well). Culture medium was changed after $24 \mathrm{~h}$ and then every 3 days. Before performing related analyses, the cell lines were cultured by decitabine $(5 \mu \mathrm{mol})$ for $4 \mathrm{~h}$.

For cDNA synthesis, RNA was treated with oligo(dT) primers (Invitrogen), $2 \mu \mathrm{l}$ RNasin (40 U/ $\mu \mathrm{l}), 8.0 \mu \mathrm{l} 5 \mathrm{X}$ reverse transcriptase buffer, $8.0 \mu \mathrm{l} \mathrm{dNTPs}$ and $2 \mu \mathrm{l}$ AMV reverse transcriptase $(5 \mathrm{U} / \mu \mathrm{l})$. The reactions were incubated for $1 \mathrm{~h}$ at $42^{\circ} \mathrm{C}, 15 \mathrm{~min}$ at $70^{\circ} \mathrm{C}$, and adjusted to a final volume of $50 \mu \mathrm{l}$. The data were normalized to $\beta$-actin reference. PIK3R2, $C C N B 1, I L 2, I L 1 B$ and $C D C 6$ were taken as examples to conduct RT-PCR validated assays and their primer sequences are listed in Table II.

For PCR amplification, the mix contained $10 \mu 1$ of $10 \mathrm{X}$ PCR buffer I, $1 \mu \mathrm{l}$ of Taq DNA polymerase (both from Invitrogen), $3 \mu \mathrm{l}$ of each forward and reverse primer and $8 \mu \mathrm{l}$ of dNTPs. Conditions were as follows: $5 \mathrm{~min}$ at $95^{\circ} \mathrm{C}$ for pre-denaturation, followed by 35 cycles of $60 \mathrm{sec}$ at $94^{\circ} \mathrm{C}$, $30 \mathrm{sec}$ at $55^{\circ} \mathrm{C}$ and $30 \mathrm{sec}$ at $72^{\circ} \mathrm{C}$, and a final 10 -min extension at $72^{\circ} \mathrm{C}$. Three replicates of the assay within or between runs were performed to assess reproducibility. Products of the PCR experiment were analyzed by $1.5 \%$ agarose gel electrophoresis and Quantity One software using a gel imaging analyzer (BioRad, Hercules, CA, USA).

\section{Results}

Detection of DEGs. Prior to the study of the DEGs between the normal controls and ovarian cancer before and after treatment with decitabine and investigation of significant genes in ovarian cancer, we designated two conditions, condition 1 (normal controls vs. ovarian cancer before treatment) and condition 2 (normal controls vs. ovarian cancer after treatment with decitabine), or in other words, condition 1 was the before treatment group and condition 2 was the after treatment group. A total of 850 and 667 DEGs were obtained from the two conditions based on SAM with $\Delta=3.600$ and 3.436, separately.

Identification of the pathogenic network. In the present study, interactions in the STRING database with a score $>0.5$ were kept as the background PPI network. With known pathogenic genes as seed genes, a network was extracted from the background PPI network, where the genes interacted with at least one seed gene. Although the genes interacting with seed genes were possibly pathogenic genes, they may also just interact with seed genes to maintain the essential biological processes for ovarian cancer. Therefore, the integration of DEGs and the network identified above helped to reduce false positives since it was believed that the expression changes of DEGs were possibly caused by the interactions with seed genes.

By mapping DEGs from condition 1 to the network extracted from background PPI network of ovarian cancer before treatment, we finally obtained a sub-network that consisted of 65 genes except 47 seed genes and 180 interactions which linked to at least one seed gene (Fig. 1). Furthermore, the genes that interacted with at least two seed genes were identified since these genes are more likely to be pathogenic genes due to their tight interactions with seed genes. As a result, 147 interactions were investigated to connect to at least two seed genes, and their interactions involved 73 genes in total, of which 41 were seed genes and the others were candidate genes; the sub-network is shown in Fig. 2 and is called pathogenic network. Notably, we found that four seed genes, KIF14, ASPM, EXO1 and RAD54L, interacted with each other and formed a clique. Therefore, these four seed genes may belong to the same complex or pathway that is involved in the pathogenic procedure. 


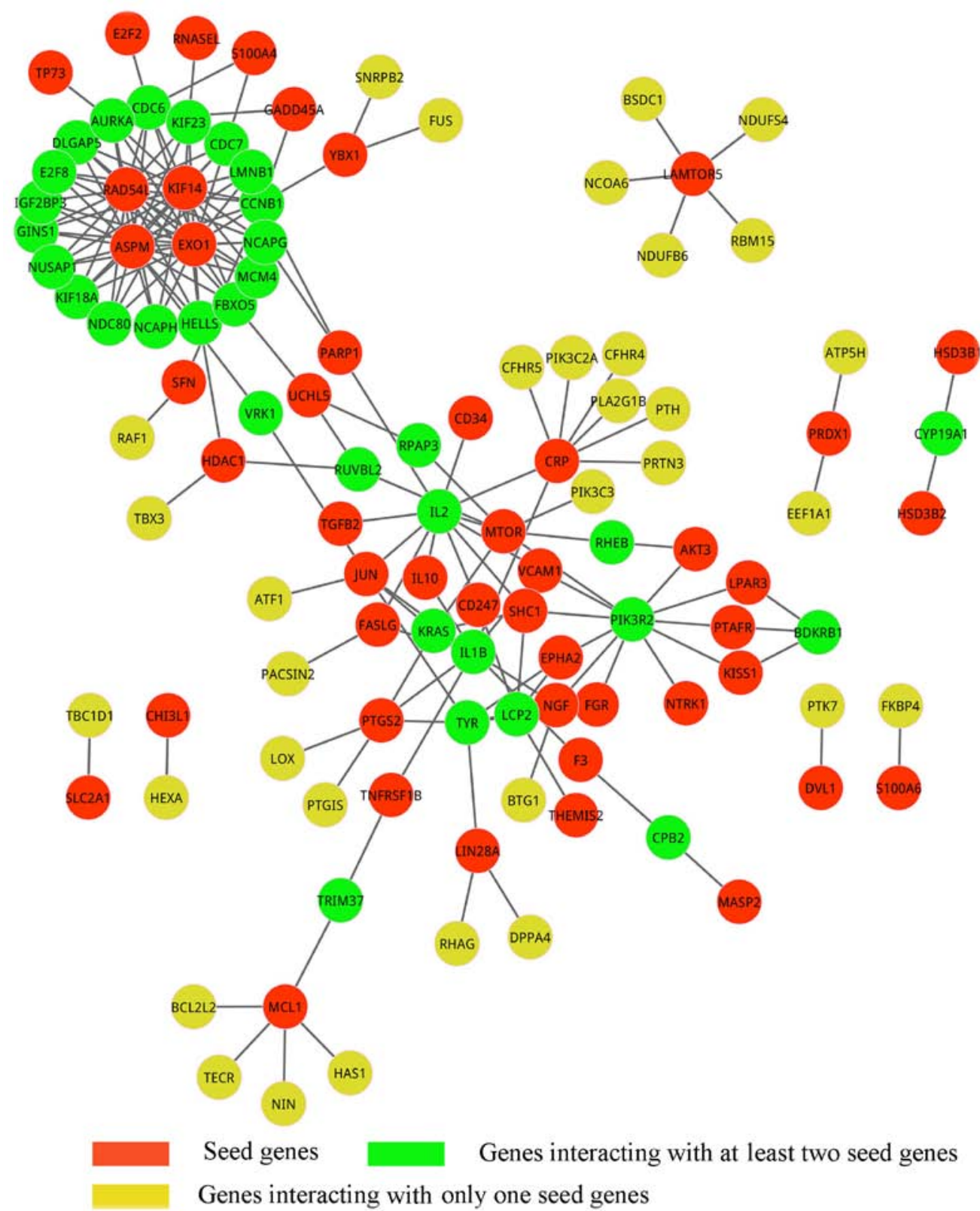

Figure 1. The sub-network of ovarian cancer before treatment. Nodes are genes, and the edge stands for the interaction between two genes. The red vertices denote seed genes from ovarian cancer, i.e. the known pathogenic genes; the green vertices stand for genes that interacted with at least two seed genes; the yellow vertices represent genes that interacted with only one seed gene.

Similarly, when changing DEGs and the background PPI network before treatment to after treatment, we obtained the sub-network (Fig. 3) and pathogenic network (Fig. 4) of ovarian cancer after treatment with decitabine. In Fig. 3, there were 83 nodes of which 39 were seed genes and 94 edges, but these genes were not entirely connected together. Discarding genes that only interacted with one seed gene, 16 candidate genes and 66 interactions were extracted from the sub-network and were formed into the pathogenic network of ovarian cancer after treatment.

Ranking of candidate genes. A total of 32 and 16 candidate genes (Tables III and IV) were identified by ranking the pathogenic genes based on the pathogenic network before and after treatment. To screen more reliable pathogenic genes, we assigned a weight to each candidate gene according to PCC, and ranked them in decreasing order. The higher weight of one gene, the more confident pathogenic gene of ovarian cancer was. For the candidate genes before treatment, IL2, PIK3R2, $I L 1 B, C D C 6$ and $C C N B 1$ possessed the top five rankings with a weight of 6.693, 6.027, 4.542, 3.890 and 3.643, respectively. The candidate genes of the after treatment group were part of that of before treatment, but their weights had great differences apart from $P I K 3 R 2$ and $C C N B 1$. The top five genes after treatment were PIK3R2, CDC7, TYR, E2F8 and CCNBI.

By comparing the two types of candidate genes, we found that the 16 candidate genes of the after treatment group were all involved in the 32 candidate genes, and the other 16 candidate genes before treatment were silenced after treatment. The silenced genes were: IL2, ILIB, CDC6, AURKA, 


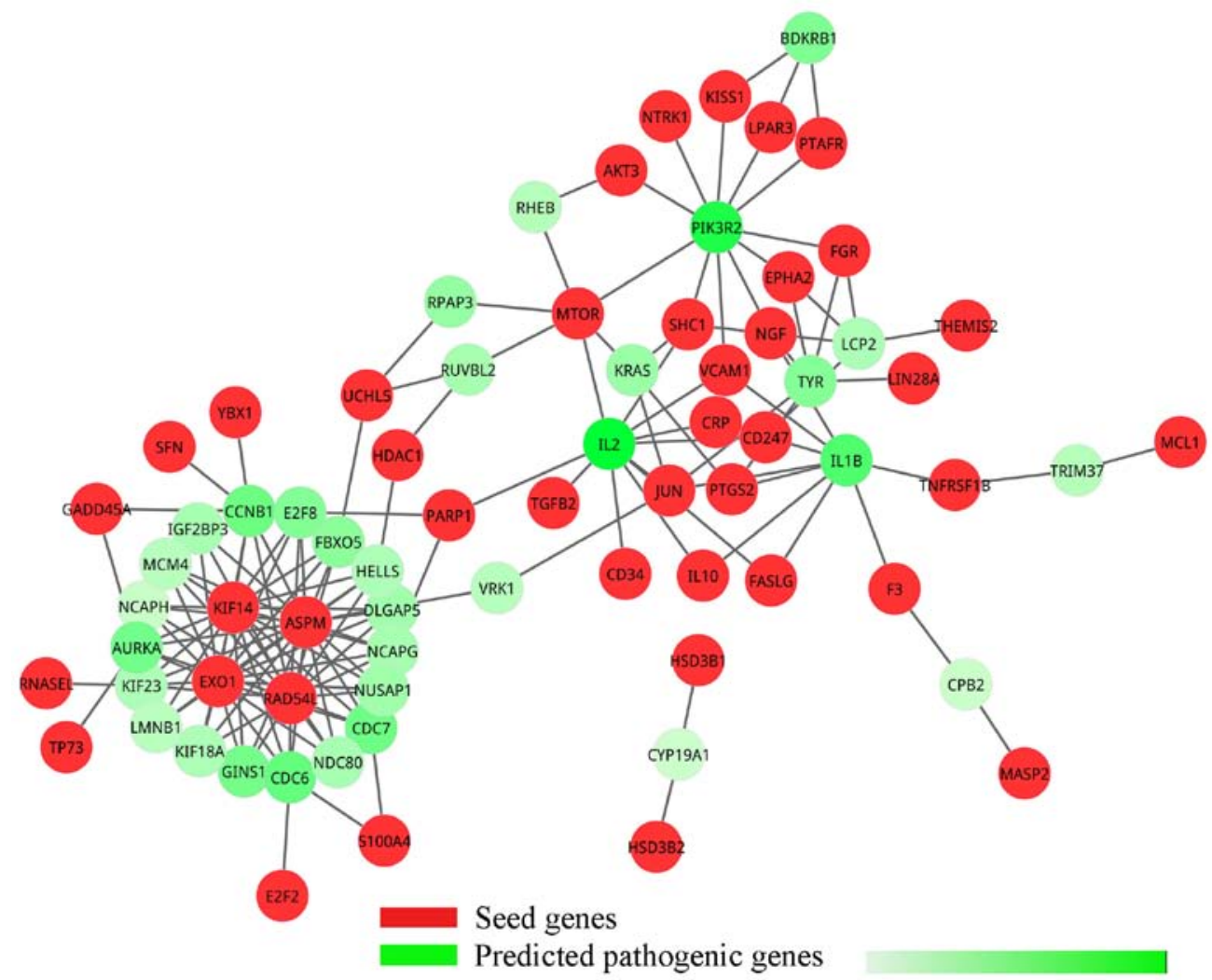

0.0

7.0

Figure 2. The pathogenic network of ovarian cancer before treatment. The red vertices denote seed genes, i.e. known pathogenic genes, the green vertices are genes that interacted with at least two seed genes, and each vertex was assigned a weight. The color bar represents the relationship between color and weight, where the deeper the color the larger is the weight.

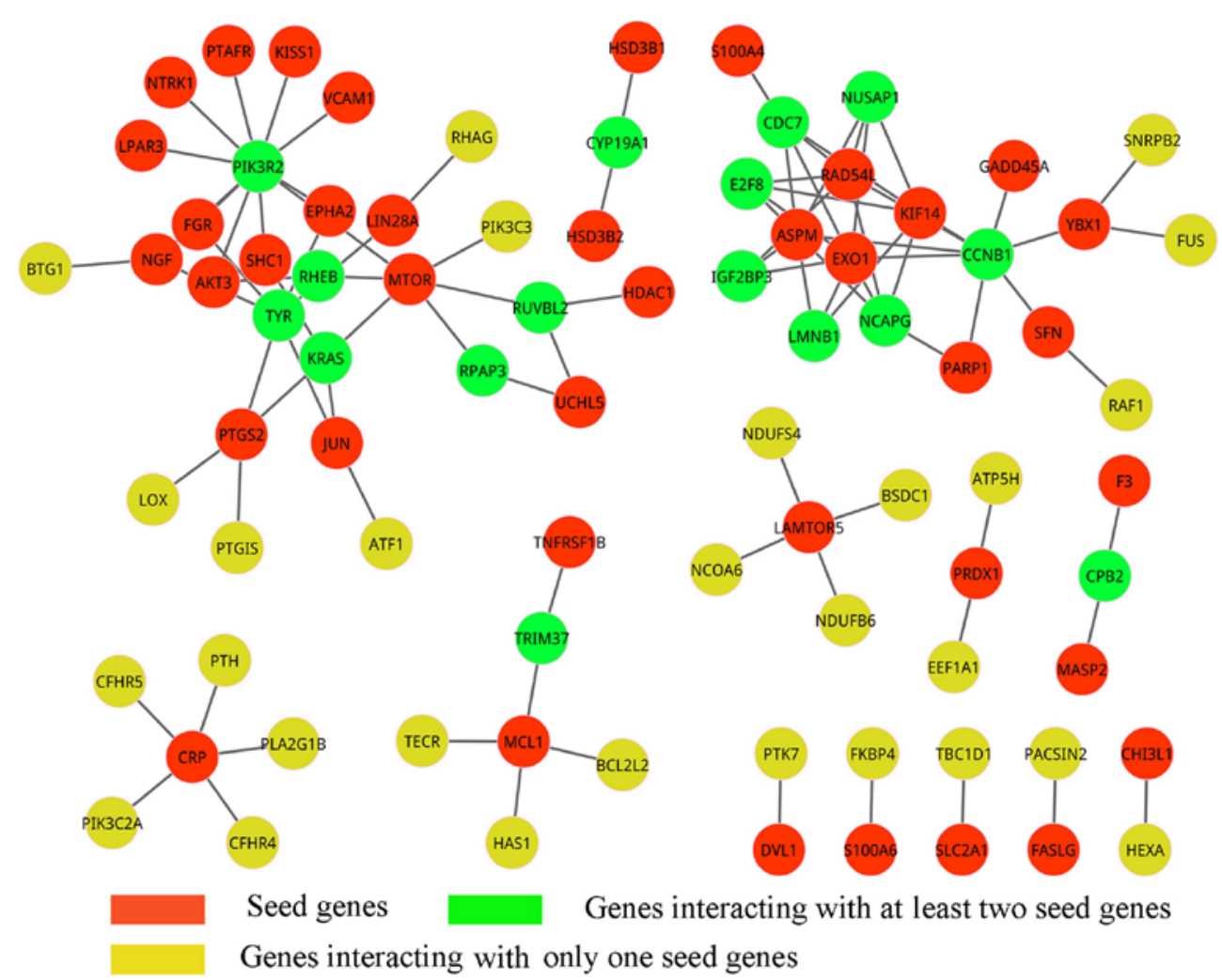

Figure 3. The sub-network of ovarian cancer after treatment with decitabine. Nodes are genes, and the edge stand for the interaction between two genes. The red vertices denote seed genes from ovarian cancer, i.e. known pathogenic genes; the green vertices stand for genes that interacted with at least two seed genes; the yellow vertices represent genes that interacted with only one seed gene. 


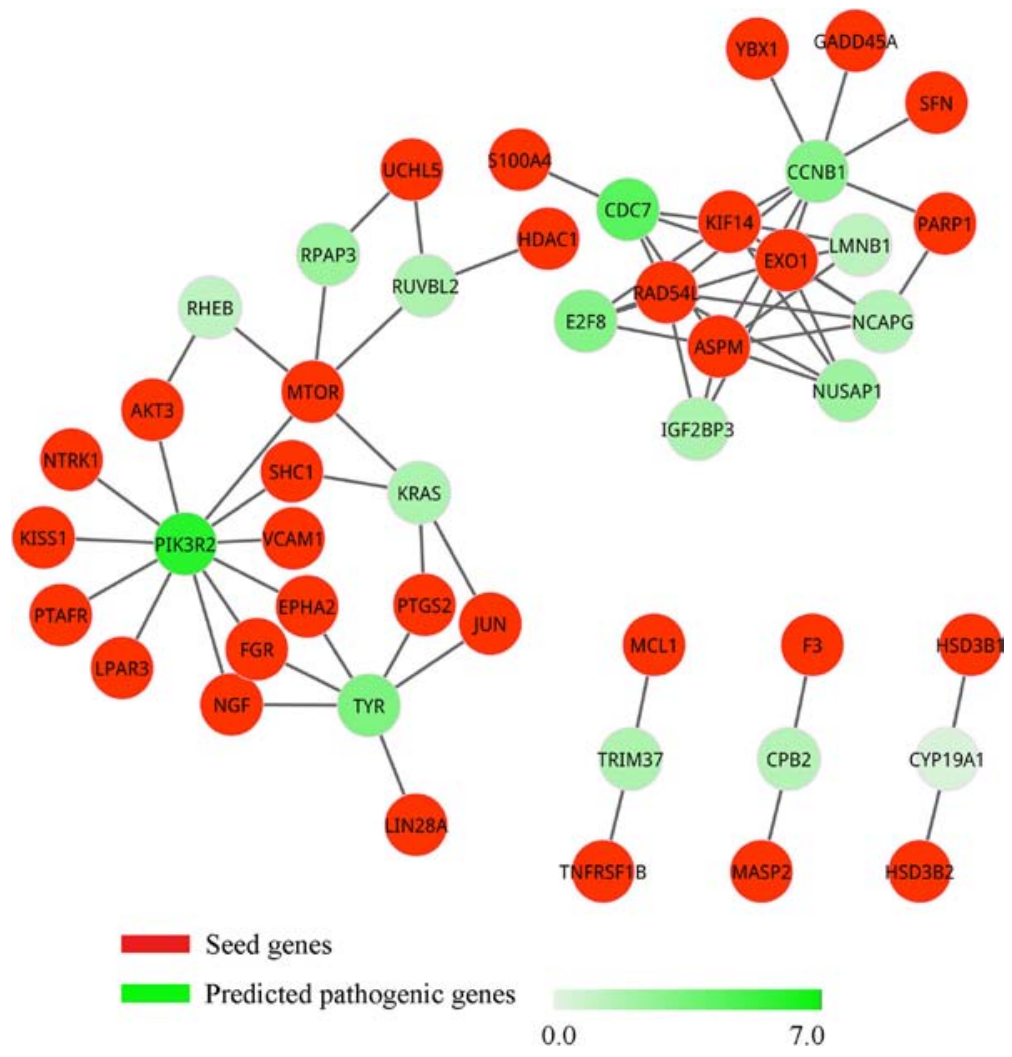

Figure 4. The pathogenic network of ovarian cancer after treatment with decitabine. The red vertices denote seed genes, i.e. known pathogenic genes, the green vertices are genes that interacted with at least two seed genes, and each vertex was assigned a weight. The color bar represents the relationship between color and weight, where the deeper the color the larger is the weight.

Table III. Weights for the candidate genes of ovarian cancer before treatment.

\begin{tabular}{clccll}
\hline Row & Node & Weight & Row & Node & Weight \\
\hline 1 & IL2 & 6.963 & 17 & NCAPG & 1.846 \\
2 & PIK3R2 & 6.027 & 18 & RUVBL2 & 1.830 \\
3 & ILIB & 4.542 & 19 & IGF2BP3 & 1.816 \\
4 & CDC6 & 3.890 & 20 & NDC80 & 1.791 \\
5 & CCNB1 & 3.643 & 21 & KIF18A & 1.680 \\
6 & CDC7 & 3.577 & 22 & KIF23 & 1.665 \\
7 & AURKA & 3.443 & 23 & HELLS & 1.624 \\
8 & GINS1 & 3.385 & 24 & LCP2 & 1.618 \\
9 & BDKRB1 & 3.026 & 25 & RHEB & 1.447 \\
10 & E2F8 & 2.904 & 26 & TRIM37 & 1.424 \\
11 & TYR & 2.854 & 27 & VRK1 & 1.377 \\
12 & FBXO5 & 2.732 & 28 & MCM4 & 1.321 \\
13 & RPAP3 & 2.386 & 29 & LMNB1 & 1.236 \\
14 & KRAS & 2.211 & 30 & NCAPH & 0.829 \\
15 & NUSAP1 & 2.080 & 31 & CPB2 & 0.777 \\
16 & DLGAP5 & 2.075 & 32 & CYP19A1 & 0.704 \\
\hline
\end{tabular}

GINS1, BDKRB1, FBXO5, DLGAP5, NDC80, KIF18A, KIF23, HELLS, LCP2, VRK1, MCM4 and NCAPH, among which IL2 changed most. The silenced genes with weight in the top five (IL2, ILIB, CDC6, AURKA and GINS1) may be more important than others for the decitabine functional process.
Table IV. Weights for the candidate genes of ovarian cancer after treatment.

\begin{tabular}{rlr}
\hline Row & \multicolumn{1}{c}{ Node } & Weight \\
\hline 1 & PIK3R2 & 6.028 \\
2 & CDC7 & 4.421 \\
3 & TYR & 3.288 \\
4 & E2F8 & 3.067 \\
5 & CCNB1 & 3.046 \\
6 & RPAP3 & 2.336 \\
7 & NUSAP1 & 2.286 \\
8 & IGF2BP3 & 1.825 \\
9 & KRAS & 1.813 \\
10 & RUVBL2 & 1.797 \\
11 & TRIM37 & 1.724 \\
12 & NCAPG & 1.590 \\
13 & CPB2 & 1.511 \\
14 & LMNB1 & 1.296 \\
15 & RHEB & 1.202 \\
16 & CYP19A1 & 0.425 \\
\hline
\end{tabular}

Identification of key genes. In the present study, several indices were utilized to investigate topological properties of candidate genes, including degree, betweenness, closeness and stress. Among the 16 common candidate genes, we removed TRIM37, CPB2 and CYP19A1 which only interacted 

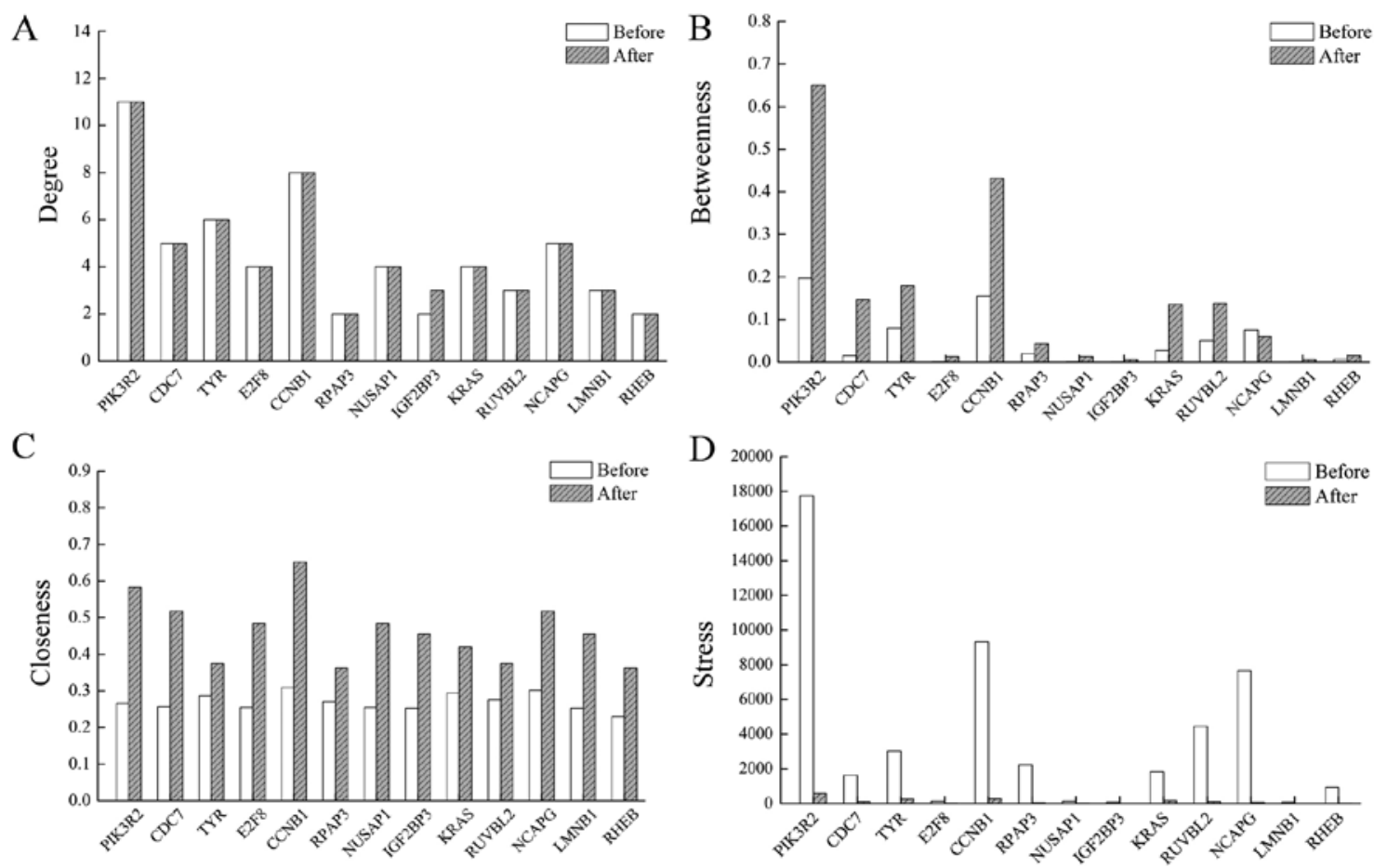

Figure 5. Topological properties of the candidate genes from ovarian cancer before and after treatment with decitabine. (A) Degree. (B) Betweenness. (C) Closeness. (D) Stress.
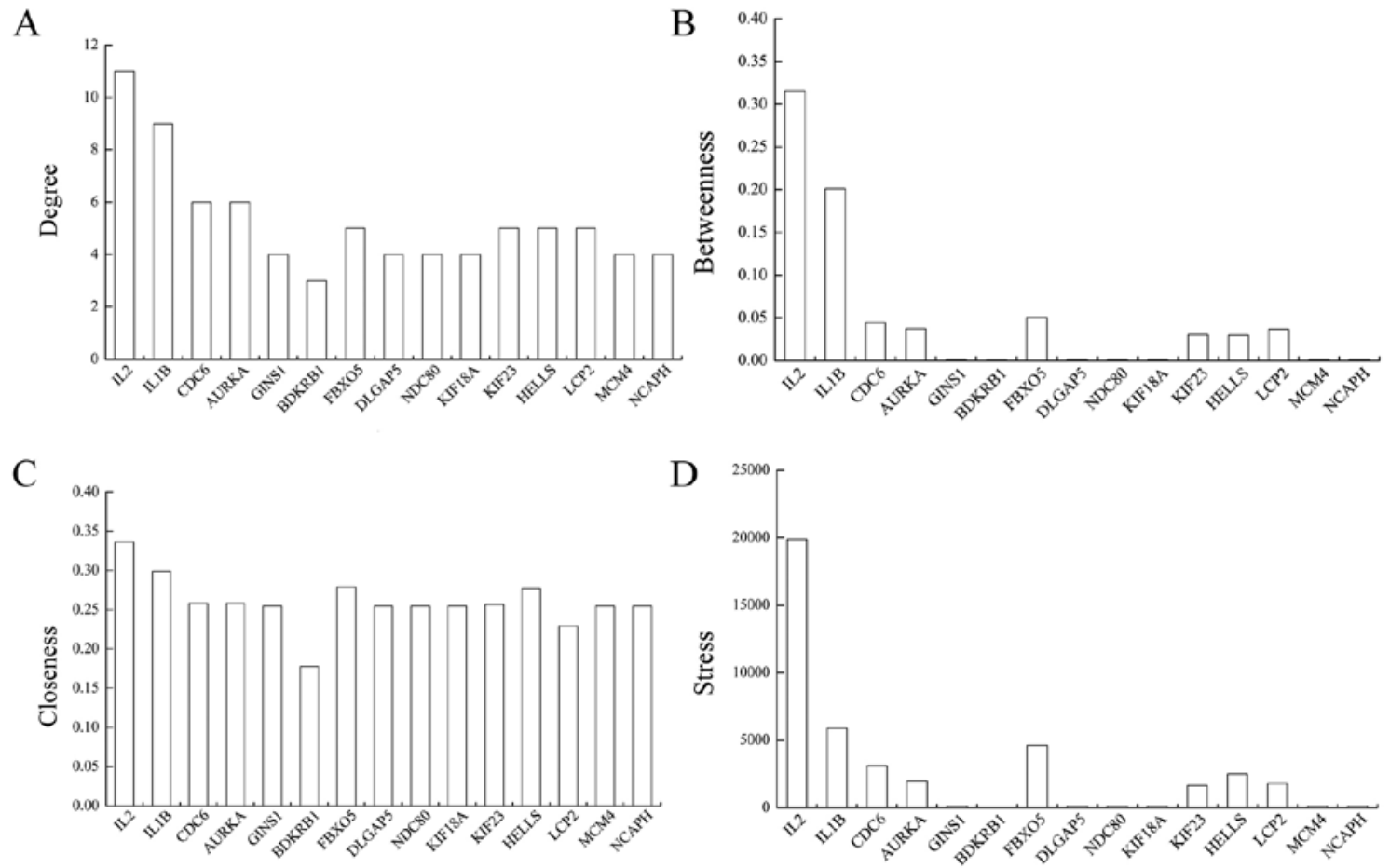

Figure 6. Topological properties of the silenced genes. (A) Degree. (B) Betweenness. (C) Closeness. (D) Stress.

with two seed genes and were not mapped main components of the pathogenic networks, and the results of the other 13 candidate genes are displayed in Fig. 5. The degree distributions for 12 candidate genes except IGF2BP3 in the before treatment group were the same as that in the after treatment group. As for betweenness and stress, $P I K 3 R 2$ and $C C N B 1$ were changed to a greater extent than the residual genes. The closeness for candidate genes in ovarian cancer before treatment was similar, but small differences were produced in after treatment. 
Table V. Pathways enriched by seed genes and candidate genes with $\mathrm{P}<0.01$.

\begin{tabular}{|c|c|c|c|}
\hline Pathway & Count & P-value & Genes \\
\hline Neurotrophin signaling pathway & 7 & $3.14 \mathrm{E}-04$ & KRAS, JUN, NTRK1,SHC1, AKT3, PIK3R2, NGF \\
\hline Cell cycle & 7 & $3.28 \mathrm{E}-04$ & CDC7, CCNB1, CDC6, HDAC1, SFN, MCM4, GADD45A \\
\hline ErbB signaling pathway & 6 & $4.76 \mathrm{E}-04$ & KRAS, JUN, SHC1, MTOR, AKT3, PIK3R2 \\
\hline Glioma & 5 & $1.272 \mathrm{E}-03$ & KRAS, SHC1, MTOR, AKT3, PIK3R2 \\
\hline $\mathrm{T}$ cell receptor signaling pathway & 6 & $1.276 \mathrm{E}-03$ & $K R A S, J U N, A K T 3, I L 2, L C P 2, P I K 3 R 2$ \\
\hline Chronic myeloid leukemia & 5 & $2.429 \mathrm{E}-03$ & KRAS, HDAC1, SHC1, AKT3, PIK3R2 \\
\hline Insulin signaling pathway & 6 & $3.413 \mathrm{E}-03$ & KRAS, RHEB, SHC1, MTOR, AKT3, PIK3R2 \\
\hline Apoptosis & 5 & $4.165 \mathrm{E}-03$ & NTRK1, IL1B, AKT3, PIK3R2, NGF \\
\hline mTOR signaling pathway & 4 & 7.113E-03 & RHEB, MTOR, AKT3, PIK3R2 \\
\hline Acute myeloid leukemia & 4 & $9.622 \mathrm{E}-03$ & KRAS, MTOR, AKT3, PIK3R2 \\
\hline
\end{tabular}

Topological properties of the silenced genes are illustrated in Fig. 6; note that VRK1 which only interacted with two seed genes was discarded. IL2 had the highest values of four topological induces, $I L 1 B$ and $C D C 6$ were next. Apart from them, degree distributions of the other silenced genes were similar, as well as closeness distribution. Meanwhile, distribution tends between betweenness and stress were almost the same.

Combining weight values and topological properties of the candidate genes and silenced genes, PIK3R2, CCNB1, IL2, $I L I B$ and $C D C 6$ were regarded as key genes for ovarian cancer treated with decitabine.

Pathway analysis. KEGG pathway enrichment analysis for the seed genes and candidate genes were carried out, and pathways with $\mathrm{P}<0.01$ which were calculated by EASE algorithm implemented in DAVID are listed in Table V. A total of 10 pathways were evaluated, of which 5 were signaling pathways (neurotrophin, ErbB $\mathrm{T}$ cell receptor, insulin and mTOR signaling pathways) and 2 (cell cycle and apoptosis) were related to cell activities. In addition, the other 3 pathways were cancer pathways (glioma, chronic myeloid leukemia and AML). The most significant 3 pathways were neurotrophin signaling pathway $(\mathrm{P}=3.14 \mathrm{E}-04)$, cell cycle $(\mathrm{P}=3.28 \mathrm{E}-04)$ and ErbB signaling pathway $(\mathrm{P}=4.76 \mathrm{E}-04)$. $P I K 3 R 2$ actively participated in 9 pathways except the cell cycle. $C C N B 1$ and $C D C 6$ were enriched in cell cycle, while IL2 mapped to T cell receptor signaling pathway.

Validation of candidate genes by RT-PCR. To study the activity and expression levels of candidate genes in ovarian cancer, we collected ovarian cancer A2780 cells before and after treatment with decitabine, and 10 ovarian cancer patient tissues to perform RT-PCR analyses. Note that the normal controls in the RT-PCR assays were para-carcinoma tissues of ovarian cancer patients. After RNA extraction, the cDNA synthesis and PCR amplification, we obtained the relative expression levels of 5 candidate genes (PIK3R2, CCNB1, IL2, $I L I B$ and $C D C 6$ ) which were taken as examples. By assessing the analysis of significance dependent on SPSS, the results are illustrated in Fig. 7. Apart from $I L 1 B$, the other four genes of ovarian cancer before treatment were significantly differentially expressed with ${ }^{*} \mathrm{P}<0.05$ compared to normal controls

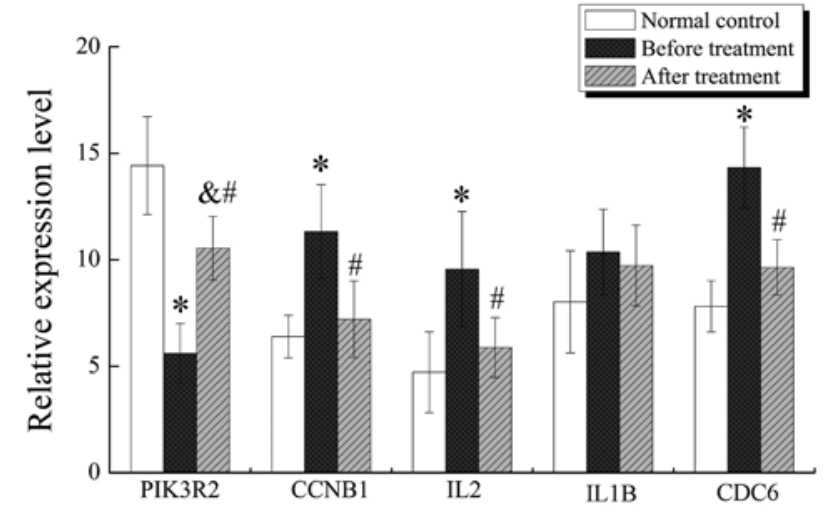

Figure 7. Relative expressions for PIK3R2, CCNB1, IL2, ILIB and CDC6. The expression of one gene in ovarian cancer before and after treatment as compared to the normal controls is indicated by its $\mathrm{P}$-value: ${ }^{*} \mathrm{P}<0.05$ indicates that the gene of ovarian cancer before treatment was significantly differentially expressed compared to normal controls; ${ }^{\&} \mathrm{P}<0.05$ indicates that the gene was significantly differentially expressed in ovarian cancer after treatment compared with the normal control; and ${ }^{\#} \mathrm{P}<0.05$ indicates that the gene was significantly differentially expressed across ovarian cancer before and after treatment.

and ovarian cancer after treatment ( $\left.{ }^{\#} \mathrm{P}<0.05\right)$. Only PIK3R2 was differentially expressed between ovarian cancer after treatment and normal controls $\left({ }^{\&} \mathrm{P}<0.05\right)$.

\section{Discussion}

In the present study, we predicted key genes associated with ovarian cancer following treatment with decitabine utilizing a pathogenic network method. The results identified 5 key genes, PIK3R2, CCNB1, IL2,IL1B and CDC6, which had high weight and good topological properties (degree, betweenness, closeness and stress) in the pathogenic network before and after treatment. In addition, these genes were validated by RT-PCR assays.

The phosphatidylinositol 3-kinase $(P I 3 K)$ enzyme is an obligate heterodimer composed of a regulatory subunit $(P I K 3 R)$ and a catalytic subunit $(P I K 3 C)$ (26). Once the interaction of $P I K 3 R$ with a variety of receptors is recruited, $P I K 3 C$ is activated through a conformational switch and produces phos- 
phatidylinositol-3,4,5-trisphosphate (PIP3), which functions as a cellular second messenger (27). PIP3 encodes kinases, of which the most important is $A K T$ that control a multitude of pathways, including cell growth, survival and metabolism (28). As a consequence, there is a close relationship between PI3K and $A K T$. It has been reported that alterations to the $P I 3 K-A K T$ signaling pathway are common in human cancer, for example, in ovarian cancer (29). We discovered that phosphoinositide3-kinase, regulatory subunit $2(P I K 3 R 2)$ and v-akt murine thymoma viral oncogene homolog 3 (AKT3) co-function in several pathways which also play significant roles in the process of ovarian cancer, such as neurotrophin signaling pathway and ErbB signaling pathway $(30,31)$. Cheung et al (32) demonstrated PIK3R2 mutations on $P I 3 K$ signaling in endometrial cancer, thus we may infer that $P I K 3 R 2$ mutations also exist in ovarian cancer.

Cyclin $\mathrm{B} 1(C C N B 1)$ is a regulatory protein involved in mitosis and the product complexes to form the maturationpromoting factor. Its transcription leading to aberrantly high levels of $C C N B 1$ throughout the cell cycle is associated with excessive hyperplasia in several human cancers (33). For example, $C C N B 1$ was found to have significant predictive power in distant metastasis-, disease- and recurrence-free survival, and overall survival of breast cancer patients (34). We found that $C C N B 1$ was overexpressed in an ovarian cancer cell line, but after decitabine treatment, its level decreased to some extent.

Interleukin 2 (IL2) is a pleiotropic cytokine produced after antigen activation and has roles in key functions of the immune system, tolerance and immunity, primarily via its direct effects on $\mathrm{T}$ cells in regards to the mediation of $\mathrm{T}$ cell growth and proliferation (35). In the present study, we found that IL2 was enriched in the $\mathrm{T}$ cell receptor signaling pathway. In ovarian tumors, myeloid cells are one of the major determinants of immune suppression, and the accumulation of these immunosuppressive activities may lead to further worsen cancer (36). Duraiswamy et al demonstrated that therapeutic pathway blockade augments other modalities of immunotherapy $\mathrm{T}$ cell function preventing immune decline in ovarian cancer (37). We may infer that IL2 had a potential role in decitabine-treated ovarian cancer patients through the medium of $\mathrm{T}$ cell.

Cell division cycle $6(C D C 6)$ is an essential regulator of DNA replication and plays important roles in the activation and maintenance of the checkpoint mechanisms in the cell cycle (38). Deregulation of CDC6 leads to aberrant DNA replication, DNA damage and genomic instability, and may even contribute to tumorigenesis (39). CDC6 has been associated with the oncogenic activities in human types of cancers, such as lung (38), breast (40) and ovarian cancer (41). For instance, Deng et al found that CDC6 was upregulated, discovered a novel regulatory signaling pathway of $C D C 6$ and provided a new potential therapeutic target for ovarian cancer patients (41). In addition, it has been suggested that a number of genes are inversely correlated with CDC6 in functional models of the ovarian cancer cell line HEYA8 (42). In the present study, we also found that CDC6 was upregulated in ovarian cancer samples.

In conclusion, we have successfully identified 5 key genes (PIK3R2, CCNB1, IL2,ILIB and CDC6) and validated them by RT-PCR. Our findings provide insight into the molecular mechanisms of decitabine treatment and may be potential pathogenic biomarkers for the therapy of ovarian cancer.

\section{Acknowledgements}

The present study received no specific grants from any funding agency in public, commercial or not-for-profit sectors.

\section{References}

1. Madathil KC, Greenstein JS, Juang KA, Neyens DM and Gramopadhye AK: An investigation of the informational needs of ovarian cancer patients and their supporters. In: Proceedings of the Human Factors and Ergonomics Society Annual Meeting. SAGE Journals 57: pp748-752, 2013. doi: $10.1177 / 1541931213571163$.

2. Network CGAR; Cancer Genome Atlas Research Network: Integrated genomic analyses of ovarian carcinoma. Nature 474: 609-615, 2011.

3. Siegel R, Naishadham D and Jemal A: Cancer statistics, 2012. CA Cancer J Clin 62: 10-29, 2012.

4. Holohan C, Van Schaeybroeck S, Longley DB and Johnston PG: Cancer drug resistance: An evolving paradigm. Nat Rev Cancer 13: 714-726, 2013.

5. Khaider NG, Lane D, Matte I, Rancourt C and Piché A: Targeted ovarian cancer treatment: The TRAILs of resistance. Am J Cancer Res 2: 75-92, 2012.

6. Liu X, Tang WH, Zhao XM and Chen L: A network approach to predict pathogenic genes for Fusarium graminearum. PLoS One 5: e13021, 2010.

7. Göhre V and Robatzek S: Breaking the barriers: Microbial effector molecules subvert plant immunity. Annu Rev Phytopathol 46: 189-215, 2008.

8. Ein-Dor L, Kela I, Getz G, Givol D and Domany E: Outcome signature genes in breast cancer: Is there a unique set? Bioinformatics 21: 171-178, 2005.

9. Zhang L, Li S, Hao C, Hong G, Zou J, Zhang Y, Li P and Guo Z: Extracting a few functionally reproducible biomarkers to build robust subnetwork-based classifiers for the diagnosis of cancer. Gene 526: 232-238, 2013.

10. Rodríguez-Paredes M and Esteller M: Cancer epigenetics reaches mainstream oncology. Nat Med 17: 330-339, 2011.

11. Ballestar E and Esteller M: Epigenetic gene regulation in cancer. Adv Genet 61: 247-267, 2008.

12. Xiang Y, Ma N, Wang D, Zhang Y, Zhou J, Wu G, Zhao R, Huang $\mathrm{H}$, Wang $\mathrm{X}$, Qiao $\mathrm{Y}$, et al: MiR-152 and miR-185 co-contribute to ovarian cancer cells cisplatin sensitivity by targeting DNMT1 directly: A novel epigenetic therapy independent of decitabine. Oncogene 33: 378-386, 2014.

13. Stephan L and Momparler R: Combination chemotherapy of cancer using the inhibitor of DNA methylation 5-aza-2'-deoxycytidine (decitabine). J Cancer Res Ther 3: 56-65, 2015.

14. Chen MY, Liao WS, Lu Z, Bornmann WG, Hennessey V, Washington MN, Rosner GL, Yu Y, Ahmed AA and Bast RC Jr: Decitabine and suberoylanilide hydroxamic acid (SAHA) inhibit growth of ovarian cancer cell lines and xenografts while inducing expression of imprinted tumor suppressor genes, apoptosis, G2/M arrest, and autophagy. Cancer 117: 4424-4438, 2011.

15. Matsumura N, Huang Z, Mori S, Baba T, Fujii S, Konishi I, Iversen ES, Berchuck A and Murphy SK: Epigenetic suppression of the TGF-beta pathway revealed by transcriptome profiling in ovarian cancer. Genome Res 21: 74-82, 2011.

16. Li J and Tibshirani R: Finding consistent patterns: A nonparametric approach for identifying differential expression in RNA-Seq data. Stat Methods Med Res 22: 519-536, 2013.

17. Amberger JS, Bocchini CA, Schiettecatte F, Scott AF and Hamosh A: OMIM.org: Online Mendelian Inheritance in Man $\left(\mathrm{OMIM}^{\circledR}\right)$, an online catalog of human genes and genetic disorders. Nucleic Acids Res 43: D789-D798, 2015.

18. Franceschini A, Szklarczyk D, Frankild S, Kuhn M, Simonovic M, Roth A, Lin J, Minguez P, Bork P, von Mering C, et al: STRING v9.1: Protein-protein interaction networks, with increased coverage and integration. Nucleic Acids Res 41: D808-D815, 2013. 
19. Benesty J, Chen J, Huang Y and Cohen I: Pearson correlation coefficient. In: Noise Reduction In Speech Processing. Springer, pp1-4, 2009. doi: 10.1007/978-3-642-00296-0_5.

20. Haythornthwaite C: Social network analysis: An approach and technique for the study of information exchange. Libr Inf Sci Res 18: 323-342, 1996.

21. Barthelemy M: Betweenness centrality in large complex networks. Eur Phys J B Cond Matter Complex Syst 38: 163-168, 2004.

22. Wasserman S: Social network analysis: Methods and Applications. Cambridge University Press, 1994. http://dx.doi. org/10.1017/CBO9780511815478.

23. Fekete SP, Kaufmann M, Kröller A and Lehmann K: A new approach for boundary recognition in geometric sensor networks. In Proc. 17th Canadian Conference on Computational Geometry. pp82-85, 2005.

24. Huang W, Sherman BT and Lempicki RA: Systematic and integrative analysis of large gene lists using DAVID bioinformatics resources. Nat Protoc 4: 44-57, 2009.

25. Wang $X$ and Simon R: Microarray-based cancer prediction using single genes. BMC Bioinformatics 12: 391, 2011.

26. Vogt PK, Hart JR, Gymnopoulos M, Jiang H, Kang S, Bader AG Zhao L and Denley A: Phosphatidylinositol 3-kinase: The oncoprotein. In: Phosphoinositide 3-kinase in Health and Disease. Springer, pp79-104, 2010.

27. Herrero-Gonzalez $S$ and Di Cristofano A: New routes to old places: PIK3R1 and PIK3R2 join PIK3CA and PTEN as endometrial cancer genes. Cancer Discov 1: 106-107, 2011.

28. Fayard E, Xue G, Parcellier A, Bozulic L and Hemmings BA: Protein kinase $\mathrm{B}(\mathrm{PKB} / \mathrm{Akt})$, a key mediator of the PI3K signaling pathway. In: Phosphoinositide 3-kinase in Health and Disease. Springer, pp31-56, 2011

29. Wu R, Hu TC, Rehemtulla A, Fearon ER and Cho KR: Preclinical testing of PI3K/AKT/mTOR signaling inhibitors in a mouse model of ovarian endometrioid adenocarcinoma. Clin Cancer Res 17: 7359-7372, 2011.

30. de Graeff P, Crijns AP, Ten Hoor KA, Klip HG, Hollema H, Oien K, Bartlett JM, Wisman GB, de Bock GH, de Vries EG, et al: The ErbB signalling pathway: Protein expression and prognostic value in epithelial ovarian cancer. Br J Cancer 99: 341-349, 2008.

31. Thiele CJ, Li Z and McKee AE: On Trk - the TrkB signal transduction pathway is an increasingly important target in cancer biology. Clin Cancer Res 15: 5962-5967, 2009.
32. Cheung LW, Hennessy BT, Li J, Yu S, Myers AP, Djordjevic B, Lu Y, Stemke-Hale K, Dyer MD, Zhang F, et al: High frequency of PIK3R1 and PIK3R2 mutations in endometrial cancer elucidates a novel mechanism for regulation of PTEN protein stability. Cancer Discov 1: 170-185, 2011.

33. Egloff AM, Vella LA and Finn OJ: Cyclin B1 and other cyclins as tumor antigens in immunosurveillance and immunotherapy of cancer. Cancer Res 66: 6-9, 2006.

34. Ding K, Li W, Zou Z, Zou X and Wang C: CCNB1 is a prognostic biomarker for ER+ breast cancer. Med Hypotheses 83: 359-364, 2014.

35. Liao W, Lin JX and Leonard WJ: Interleukin-2 at the crossroads of effector responses, tolerance, and immunotherapy. Immunity 38: 13-25, 2013.

36. Wilke CM, Kryczek I and Zou W: Antigen-presenting cell (APC) subsets in ovarian cancer. Int Rev Immunol 30: 120-126, 2011.

37. Duraiswamy J, Freeman GJ and Coukos G: Therapeutic PD-1 pathway blockade augments with other modalities of immunotherapy T-cell function to prevent immune decline in ovarian cancer. Cancer Res 73: 6900-6912, 2013.

38. Zhang X, Xiao D, Wang Z, Zou Y, Huang L, Lin W, Deng Q, Pan H, Zhou J, Liang C, et al: MicroRNA-26a/b regulate DNA replication licensing, tumorigenesis, and prognosis by targeting CDC6 in lung cancer. Mol Cancer Res 12: 1535-1546, 2014.

39. Blow JJ and Gillespie PJ: Replication licensing and cancer - a fatal entanglement? Nat Rev Cancer 8: 799-806, 2008.

40. Booher K, Lin DW, Borrego SL and Kaiser P: Downregulation of Cdc6 and pre-replication complexes in response to methionine stress in breast cancer cells. Cell Cycle 11: 4414-4423, 2012.

41. Deng Y, Jiang L, Wang Y, Xi Q, Zhong J, Liu J, Yang S, Liu R, Wang J, Huang M, et al: High expression of CDC6 is associated with accelerated cell proliferation and poor prognosis of epithelial ovarian cancer. Pathol Res Pract: Sep 18, 2015 (Epub ahead of print). pii: S0344-0338(15)30014-5. doi: 10.1016/j. prp.2015.09.014.

42. Creighton CJ, Hernandez-Herrera A, Jacobsen A, Levine DA, Mankoo P, Schultz N, Du Y, Zhang Y, Larsson E, Sheridan R, et al; Cancer Genome Atlas Research Network: Integrated analyses of microRNAs demonstrate their widespread influence on gene expression in high-grade serous ovarian carcinoma. PLoS One 7: e34546, 2012. 